\title{
LA INVESTIGACIÓN SOCIAL CONTEMPORÁNEA: DIFICULTADES, PROBLEMAS Y DESAFÍOS
}

\section{CONTEMPORARY SOCIAL RESEARCH: DIFFICULTIES, PROBLEMS AND CHALLENGES}

\section{Mirella Del Pilar Vera-Rojas'}

\section{Luis Fernando Chávez Vera²}

\section{Luis Antonio Vera Rojas ${ }^{3}$}

Universidad Nacional de Chimborazo,

Escuela Superior Politécnica de Chimborazo, Instituto Superior Tecnológico "San Gabriel", Riobamba, Ecuador

$1 \quad$ mire6.unach@yahoo.com Profesional en Filosofía y Ciencias Socio - Económicas y Doctora en Planeamiento y Diseño Curricular de la Universidad Nacional de Chimborazo; Magister en Docencia Universitaria e Investigación Educativa de la Universidad Nacional de Loja, y Doctora Ph.D en Ciencias Pedagógicas por la Universidad de La Habana - Cuba. Vicerrectora Académica del Instituto Tecnológico Superior San Gabriel de la ciudad de Riobamba 2004-2010 y Docente Investigadora de la Facultad de Ciencias de la Educación, Humanas y Tecnologías de la UNACH. https:// orcid.org/0000-0001-6896-1391

2 chavezveraluisfernando@gmail.com Licenciado en Laboratorio Clínico e Histopatológico de la Universidad Nacional de Chimborazo, y Máster Propio en Ciencias Forenses y Criminalística de la Universidad de Valencia España. Profesor y Coordinador del Departamento de Vinculación de la Carrera de Enfermería del Instituto Superior Tecnológico "San Gabriel" de la ciudad de Riobamba. https:// orcid.org/0000-0002-9813-3404

$3 \quad$ Ivera@espoch.edu.ec Doctor en Matemática y Magister en Matemática Aplicada Mención Modelación Matemática y Simulación Numérica de la Escuela Superior Politécnica de Chimborazo. Director y Profesor de la Carrera de Física y Matemática de la Facultad de Ciencias de la ESPOCH en el que ha dictado las cátedras de Cálculo Matemático y Estadística Descriptiva e Inferencial. https:// orcid.org./0000-0002-7087-8447

\section{RESUMEN}

El artículo propuesto centra su análisis en la reflexión de los problemas, dificultades y desafíos de la investigación social contemporánea, para lo cual fue necesario partir del debate histórico entre métodos de investigación cuantitativa y cualitativa y la inserción del positivismo en las prácticas de los investigadores, llegando a señalar sobre la bibliografía revisada de varios autores y autoras, propuestas de superación de este debate mediante la aplicación de "multimétodos", de "métodos mixtos" o de la "triangulación metodológica", dando lugar a diversas perspectivas de la investigación social y una serie de consecuencias en torno a su diseño, caracterizado por su flexibilidad, que 
adquiere confiabilidad y validez a través de metodologías como la "vigilancia metodológica", "hacer observable lo inobservable" que junto con la "Teoría Fundamentada" permite a la metodología cualitativa superar la fragmentación en el análisis de los datos y al mismo tiempo conceptualizar y clasificar en forma más clara los datos provenientes de la base empírica. Posteriormente se analiza cómo superar el sentido común de los invetigadores a través de la teoría, tan necesaria para explicar la parte subyacente en las obervaciones sobre el mundo, donde la articulación teoría, método/técnica y base empírica es fundamental al momento de analizar los datos de la realidad que no se recogen, sino que se los producen a través del encuentro entre investigador e investigado, quienes construyen cooperativamente el conocimiento. Seguidamente se presenta una crítica a la investigación positivista imperante en el contexto ecuatoriano, que junto a políticas erradas entorpecen y muchas de las veces impiden el desarrollo de investigaciones en el campo social. Finalmente, se plantean desafíos a nuestras propias prácticas investigativas frente a los giros epistémicos de lo feminista, lo ambiental, lo decolonial, de la hermenéutica y de la complejidad; cerrando el análisis momentáneamente con la importancia de promover procesos éticos en la investigación social.

PALABRAS CLAVE: Investigación social cualitativa, multimétodos, métodos mixtos o triangulación metodológica y teoría fundamentada, investigación cuantitativa positivista.

\section{ABSTRACT}

The proposed article focuses its analysis on the reflection of the problems, difficulties and challenges of contemporary social research, for which it was necessary to start from the historical debate between quantitative and qualitative research methods and the insertion of positivism in the practices of researchers. coming to point out on the revised bibliography of several authors, proposals for overcoming this debate through the application of "multimethods", "mixed methods" or "methodological triangulation", giving rise to different perspectives of social research and a series of consequences around its design, characterized by its flexibility, which acquires reliability and validity through methodologies such as "methodological surveillance", "making the unobservable observable" which together with "Grounded Theory" allows qualitative methodology overcome fragmentation in data analysis while conceptualizing and classifying $r$ more clearly the data from the empirical base. Subsequently, it is analyzed how to overcome the common sense of the investigators through the theory, so necessary to explain the underlying part in the observations about the world, where the articulation of theory, method / technique and empirical basis is fundamental when analyzing the data. of reality that are not collected, but are produced through the encounter between researcher and researched, who cooperatively build knowledge. Next, a critique of the prevailing positivist research in the Ecuadorian context is presented, which together with wrong policies hinder and many of the times impede the development of research in the social field. Finally, challenges are posed to our own investigative practices in the face of the epistemic turns of the feminist, the environmental, the decolonial, the hermeneutic and the complexity; closing the analysis momentarily with the importance of promoting ethical processes in social research.

KEY WORDS: Qualitative social research, multimethods, mixed methods or methodological triangulation and grounded theory, positivist quantitative research.

\section{DESARROLLO}

A lo largo de la historia de la ciencia, se ha debatido y se continua debatiendo acerca de la validez y rigurosidad de los métodos de la 
investigación cuantitativa, frente a los métodos de la investigación cualitativa, llegando a establecer que los métodos cuantitativos liderados por el método científico experimental propio de las ciencias duras, se caracterizan por controlar, medir y manipular variables que están en juego en las hipótesis que se plantean; mientras que los métodos cualitativos propios de las ciencias sociales liderados por el paradigma heurístico - interpretativo y vinculados con la etnografía, la fenomenología, la sociología y la teoría crítica permiten describir vivencias y características de los sujetos que se investiga.

Dentro de esta línea de pensamiento es importante reconocer los límites que tiene el método experimental positivista, tanto por la imposibilidad de asumir el presupuesto de que todos los objetos de un mismo tipo son iguales y se comportan de la misma manera, lo que equivaldría a decir como bien lo expresa Piovani (2018) que todas las familias son iguales y que tienen las mismas respuestas a los mismos estímulos, y por otro lado está también la imposibilidad operativa y ética de controlar, medir y manipular variables, específicamente las variables que están en juego en las hipótesis que se plantean.

Las críticas al positivismo van entonces dirigidas en torno a que: la realidad es concebida como externa, predefinida y predeterminada, cuando desde el punto de vista cualitativa se la concibe como algo dinámico, construido y por lo tanto algo que se puede modificar, intervenir e incluso también transformar la realidad existente (Marradi, Archenti y Piovani, 2007); a la defensa de una unicidad (monismo) del método para todos los objetos y disciplinas tomando como modelo al de las ciencias físicas y naturales reduciendo la complejidad de lo social; la dificultad de uso del método experimental para el estudio de fenómenos sociales; la no contemplación de la existencia de diferentes planos en la realidad (objetada desde el paradigma de la complejidad entre otros); la invisibilización de la singularidad de la experiencia del/la observador/a pensándolo/a desde una exterioridad; la limitación en sus alcances dado que los hechos sociales no pueden reducirse a leyes universales por ser hechos de cultura, entre otras, razón por la cual la aspiración positivista en investigación social es irrealizable, por to que dentro del debate científico cuantitativo y cualitativo, las ciencias sociales plantean la necesidad de desarrollar otros métodos que logren controlar impersonalmente las hipótesis en el plano de contrastación empírica (Marradi y Piovani, 2002).

Frente a esta dicotomía, acertadamente Piovani (2018) señala que en el contexto actual, al menos en el plano retórico, hay muchos autores $y$ autoras que plantean que estamos en una fase inicial de superación de este debate, y que este tiene que ver, o con la mera convivencia de métodos cuantitativos y cualitativos que se los acepta en un plano de igualdad desde el punto de su valor científico, o con el hecho de complementar lo cuantitativo y lo cualitativo en una misma investigación, articularlos, integrarlos, o incluso en una posibilidad de trascendencia, algo que nos permita superar completamente nuestras referencias a lo cuantitativo y lo cualitativo.

Esta nueva fase del debate tiene mucho que ver, con lo que conocemos en la actualidad como las propuestas de "multimétodos", de "métodos mixtos" o de "triangulación metodológica" que son distintas formas de proponer la complementariedad o la articulación o la integración de los métodos cuantitativos y cualitativos, por lo que se debe prestar atención a las diferencias entre multimétodos y triangulación metodológica (Pavioni, 2018).

Esta perspectiva de la investigación social tiene desde el punto de vista del diseño una serie de consecuencias muy importantes, la primera es 
que habilita el pluralismo metodológico, no hay por lo tanto una única forma de diseño, ni un único método para construir conocimiento sobre la realidad observable; en segundo lugar, que le confiere a los procesos de investigación y a su diseño cierta flexibilidad, cierta capacidad de ser redefinidos en el mismo proceso de investigación; y en tercer lugar, es que permite recuperar el importante rol del conocimiento personal y tácito en la investigación científica, porque los métodos de investigación y especialmente las técnicas de investigación remiten a conocimientos procedimentales muy articulados, muy establecidos que forman parte de un aspecto fundametal del proceso de investigación (Piovani, 2018).

De ahí que:

"La flexibilidad de los diseños cualitativos no contiene reglas fijas ni procedimientos únicos para la producción y análisis de datos; sin embargo, no queda librada la tarea de investigación solo al criterio personal, el cual puede basarse en el sentido común, muchas veces alejado del método científico, sino que un amplio conjunto de criterios metodológicos orienta y pautan las decisiones" (Cohen y Seid, 2019, p. 203).

Tales criterios metodológicos, constituyen para Cohen y Gómez Rojas (2019), la "vigilancia metodológica" que debe estar presente en todo proceso de investigación trátese del problema que sea, y por ende atravesado por las cuatro dimensiones que la constituyen como es la temporalidad, fuente de información, perspectiva y lógica experimental que "según cómo se presenten, aportan metodológicamente a la estrategia de la investigación"(Cohen y Gómez Rojas, 2019, p. 254), otorgando confiabilidad y validez a todo el trabajo de investigación.
Como se denota, durante la etapa de producción y análisis de datos tal como lo sostienen los autores antes mencionados, diversas estrategias metodológicas en la investigación social ayudan a evitar la manera fragmentaria y naturalista ingenua de interpretar los datos criticada por Coffey y Atkinson (2003) y Athkinson y Delamont (2004), entre ellas la Teoría Fundamentada que tiene como propósito, no solo el registro de un fragmento textual a través de la codificación, sino la generación de teoría, en donde, la codificación de las categorías para conceptualizar y clasificar los fenómenos estudiados, quedarán abiertas para ir descomponiendo minuciosamente los instrumentos de registro utilizados y a manera de prueba y error ir construyendo las categorías necesarias durante el análisis de los datos (Cohen y Gómez Rojas, 2019).

Otra estrategia metodológica que ayuda a corresponder lo conceptual y lo empírico dentro de la investigación en las ciencias sociales, pertimiento hacer observable lo inobservable, es la propuesta desarrollada por Raymond Boudon y Paul Lazarsfeld (1973), para lo cual es necesario tomar un concepto desde una teoría, dimensionarlo, crear indicadores y con los indicadores construir índices, con lo que obtendremos una expresión empírica, una medición, pero no como una asignación de números a las cosas, tal como lo haría la concepción positivista, sino como una definición, puesto que medir en ciencias sociales es hacer observable conceptos inobservables.

Desde esta perspectiva crítica de análisis, la investigación social cualitativa tiene un potencial muy importante para el estudio de la sociedad. Es un tipo de metodología que ha ganado espacio en los círculos académicos. Entendemos que una de las tareas cruciales de este tipo de investigación, y que lo hemos venido abordado en párrafos anteriores, es el manejo de la información que nos permite la construcción y análisis de datos, (Schettini, 
2015), y que constituye el punto central en la producción del conocimiento.

Por ello, la importancia y necesidad de que los investigadores superemos las ideas de sentido común, rompamos esquemas mentales, cosmovisiones, e imposiciones ideológicas y dogmáticas que impiden interpretar objetivamente la realidad, para fundar un problema de investigación social sobre la base de la teoría científica, entendida esta como la luz que ilumina y guía todo proceso investigativo, puesto que las teorías a decir de Stinchcombe (1968), son tan necesarias para explicar la pauta subyacente en las observaciones sobre el mundo, puesto que la fundación de un problema y el desarrollo de una investigación veraz tal como lo plantea Cohen y Gómez Rojas (2019) depende de la articulación entre: teoría, método/ técnica y base empírica.

Estas y otras razones influyeron para que a lo largo de este análisis, se reflexione desde una mirada superadora del positivismo que comprende que el objeto está allí afuera para ser analizado, y se destaca, la importancia de la teoría presente en todo el proceso de investigación, así como también en el plantemiento de que los datos no se "recogen, o se recolectan" sino que se "construyen" desde el inicio del proceso, puesto que:

"Cuando afirmamos que la investigación social es un proceso teórico y empírico a la vez, estamos señalando que el conocimiento producido en la investigación resulta de la interacción -y muchas veces de la tensión- entre el conocimiento acumulado expresado en conceptos, la relación entre ellos (definiciones, hipótesis, afirmaciones, etcétera), y un conjunto de hechos que pretendemos conocer, interpretar, analizar y dar cuenta de ellos en el marco (a partir) de ese conocimiento acumulado. De esta interacción se espera la construcción de un nuevo conocimiento que contendrá estos nuevos hechos, los cuales dejarán de ser lo que son para transformarse en datos, entendidos como representación teórica de los hechos" (Cohen y Gómez Rojas, 2019:p. 29-30).

Siguiendo dentro de esta misma línea conceptual, Cohen y Gómez Rojas (2019) critican la homologación del hecho con dato desde la perspectiva empirista, puesto que reproduce la disociación entre teoría y método, porque supone que el abordaje del hecho es exclusivamente una cuestión de método y técnica. Desde esta perspectiva empirista, el investigador asume un rol pasivo y dependiente del hecho como generador de teoría.

Con el afán de superar esta tendencia positivista Cornejo (2011) plantea que:

"En el escenario de la investigación social cualitativa se ha ido materializando el interés contemporáneo por temáticas del sujeto y de lo subjetivo como objetos de estudio científico. La investigación se asume entonces como un evento dialógico, contextual y situado, donde ocurre el significado y donde es posible construir conocimiento. Así, el encuentro entre investigador e investigado es considerado como el lugar a partir del cual es posible construir este conocimiento" (p. 1).

Aquí entra en juego el involucramiento del sujeto con el objeto de estudio, pero ¿Qué es involucrarse? ¿Es algo que interfiere con la investigación? $O$ por el contrario ¿Es algo positivo para el proceso de investigación al generarse un vínculo o una empatía con el sujeto/ objeto de estudio? Las y los investigadores podemos involucrarnos con la problemática de estudio, e incluso podemos estar afectados directamente por ella, pero eso no implica que 
actuemos desde el sentido común, se trata de cuestiones distintas (Gorostegui, 2020). Ese involucramiento o compromiso no debería orientar las preguntas-problema ni el análisis de los datos, sino que estos procesos deberían emerger de la mencionada articulación entre la teoría, el método y la base empírica tal como lo sostiene Cohen y Gómez Rojas (2019).

Además del involucramiento entre sujeto $y$ objeto de estudio, es importante determinar la mirada del sujeto dentro de los procesos de investigación social, que luego de deliberar en los foros se señala que si bien es casi imposible "despojarse de la propia mirada" y dejar de "ponerse en el lugar del otro", pero eso no quita que debemos ser conscientes de esa mirada y esa empatía para poder distinguirla y desarmarla a partir de los conocimientos que vamos construyendo desde la teoría y el método.

Al respecto, Cohen y Gómez Rojas (2019) nos dicen que "nuestras intervenciones son intervenciones comprometidas, atravesadas por nuestros posicionamientos; por lo tanto, es imprescindible ser conscientes de desde dónde observamos y debemos hacer visibles tales perspectivas" (p. 83). Además, el "ponerse en el lugar del otro" como investigadores e investigadoras puede traducirse en comprender al sujeto-objeto de estudio, interpretar sus decires, sus percepciones, opiniones y experiencias sobre una determinada problemática, y al mismo tiempo dar lugar a su perspectiva para interpretarla en función de nuestros objetivos y la teoría que nos respalda.

En cuanto al encuentro entre investigador e investigado dentro de la investigación cualitativa, comparto el criterio de que su accionar va mucho más allá de la producción de datos, puesto que permite la construcción cooperativa del conocimiento, al reconocer que ambos comparten la misma dignidad, llegando entonces al verdadero significado de lo esencial o dignitario, por eso que en la investigación cualitativa y especialmente en el enfoque epistemológico del sujeto conocido fundamento ontológico y epistemológico de la metodología cualitativa de hoy, se objeta la validez de la investigación que no dé cuenta de esta interacción (Vasilachis, 2009).

Por lo tanto, el diálogo entre los sujetos que participan en la investigación cualitativa -investigador e investigado- es sustancial dentro de esta nueva propuesta epistemológica, puesto que al permitir la pluralidad de interpretaciones, perspectivas y prácticas entre los sujetos, además de enriquecer los resultados de la investigación, se demuestra que hay otros criterios de validez en la producción del conocimiento, puesto que conocer a una persona supone un cómo, un método distinto al que se utiliza para conocer al qué objeto en una investigación cuantitativa. De ahí que es importante reconocer que la investigación cualitativa y la investigación cuantitativa tienen distintos diseños y también distintos procesos.

Lamentablemente en nuestro medio profesional y concretamente en el contexto ecuatoriano, se sigue empleando predominantemente en las investigaciones el modelo positivista -que resume todo a lo medible y cuantificable-, tanto en las investigaciones de pregrado como de posgrado sean estas de corte social o natural, lo que está impidiendo la producción de nuevas teorías a partir de nuestro contexto y de nuestras realidades, tan necesarias hoy por hoy, si queremos descolonizar nuestro pensamiento, nuestras prácticas investigativas y empezar a producir ciencia, tendiente a resolver problemas que nos atañen desde nuestras particularidades.

Otro aspecto importante a considerar dentro de esta reflexión, tiene que ver con los desafíos a los que se debe enfrentar nuestras propias prácticas investigativas en el contexto latinoamericano, ante los giros epistémicos de lo feminista, lo ambiental, lo decolonial, de la hermenéutica y de la complejidad emergente Alvarado (2018) como 
punto de partida y análisis, reflexión, crítica y autocrítica para rescatar nuestra voz, y preservar el planeta para convivir armoniosamente con él, son grandes, y muchos de ellos salen incluso de nuestras manos, puesto que quienes hacemos investigación social, sobre todo en el campo educativo, seguimos luchando ante la poca importancia que le dan a investigaciones de este tipo, donde resulta más importante asignar presupuesto para determinar la calidad de ladrillo para la venta, que para investigar los problemas que aquejan a la educación, por lo que hace falta "someter a la autocrítica nuestro pensamiento y actitud en la práctica de la ciencia, revisar y superar prejuicios como la creencia en la verdad única, o lo que llama Steriner la nostalgia de los absolutos, que nos impiden una capacidad de escucha desde el pluralismo y la inter y transdisciplinariedad" (Alvarado, 2020, p. $1)$.

Otro de los desafíos coyunturales será entonces promover la cultura de investigación en estudiantes y profesores y empezar a proponer trabajos innovadores desde el pluralismo y la inter y transdisciplinariedad que permita el análisis de un mismo fenómeno desde varias perspectivas y enfoques, para lo cual es importante la actualización y perfeccionamiento teórico y práctico de los investigadores en nuevas formas y maneras de hacer investigación, como las que hemos estudiado a lo largo de esta especialización, y superar nuestras limitaciones conceptuales para estar en la posibilidad de romper la rigidez de las posturas epistemológicas paradigmáticas del objetivismo, subjetivismo y constructivismo, y dejarlas de considerar como la única forma de hacer ciencia (De Sousa, 2008; Piovani 2018).

Además, en el contexto ecuatoriano, se necesita promover cambios sustanciales en la normativa de investigación impuesta en las instituciones de educación superior, puesto que se han convertido en trabas al momento de hacer investigación, así como también proponer investigaciones que permitan reflexionar sobre nuestra propia práctica pedagógica y didáctica y a través de ello romper nuestros esquemas mentales impuestos y creados para mantener la dominación, y empecemos a pensar descolonizadamente, fuera de lo establecido, para dejar de ser objetos y pasar a ser sujetos de nuestra propia historia, replanteando nuestras prácticas epistemológicas y metodológicas, nuestras prácticas pedagógicas y didácticas valorando lo nuestro sin desconocer lo global, puesto que el pensar en lo propio implica también pensar con lo de los otros y por ende construirnos intersubjetivamente y de la mejor manera, donde se escuche las voces de todos y todas (De Sousa, 2008).

Finalmente se aborda el tema de la ética en la invetigación social, para lo cual desde la bibliografía estudiada y el debate en los foros se llega a establecer que una "Comisión de Ética de la Investigación en Ciencias Sociales" es importante y necesaria, pero debería tener como objetivos tanto la elaboración de un conjunto de códigos éticos, como también de objetivos que permitan evaluar en forma integral, cada una de las etapas de las investigaciones propuestas, con lo que de alguna manera se vendría a superar algunos obstáculos ocasionados por los propios investigadores mencionados por Tapia (1995) tales como:

"Falta de honradez en el trabajo científico; falta de conocimiento de la metodología de investigación; carencia de espíritu de investigación; y parcialidad en el desarrollo de la investigación" ( $p$. 33-36), así como también se podría hacer frente a obstáculos de carácter sociocultural que continúan presentes al momento de hacer investigación, no solo en el campo de las Ciencias Sociales tales como "el etnocentrismo, el subjetivismo, el autoritarismo, el 
dogmatismo, el impresionismo, los estereotipos y el especialismo" (Tapia, 1995, p. 33-36).

En tal virtud, los investigadores sociales debemos tener la consciencia de que los comités o comisiones de ética tienen la tarea de evaluar los diseños de investigación con el fin de proteger la dignidad de las personas que participan o participarían de la investigación, más allá de las metas propiamente del estudio (Achío 2003). Por otro lado, y concordando con Pattullo, si se prepara a los sujetos que participan en investigaciones, pueden también asumir razonablemente las responsabilidades para su propia protección (1982:388 en Achío 2003:91).

\section{A MODO DE CIERRE}

Las dicotomías entre las orientaciones metodológicas cuanti-cualitativas dentro de la investigación pueden ser superadas desde la aplicación de "multimétodos", de "métodos mixtos" o de "triangulación metodológica" que son distintas formas de proponer la complementariedad, articulación o la integración de los métodos cuantitativos y cualitativos dentro de la investigación.

Se destaca dentro de la investigación social el paso del sentido común hacia un conocimiento veraz y válido sobre la base de la teoría, método/ técnica y base empírica, propuesta por diversos autores y autoras, estableciendo su ruptura al abordarlos científicamente.

La triangulación metodología abre horizontes para pensar en futuras investigaciones que permitan integrar y converger en un mismo objeto de estudio resultados cuantitativos al lenguaje cualitativo y viceversa, manteniendo una vigilancia metodológica y una actitud de reflexividad (Santos y Pi Puig, 2018) cuando utilizamos métodos mixtos, para no caer en la trampa que señala Bryman (2006) de la superación de lo cuantitativo o cualitativo.
La Teoría Fundamentada permite a la metodología cualitativa superar la fragmentación en el análisis de los datos y al mismo tiempo conceptualizar y clasificar en forma más clara los datos provenientes de la base empírica.

La propuesta metodologica de Lazarsfeld desarrollada sobre cuatro operaciones básicas como la descripción literaria del concepto, la identificación de sus dimensiones, la elección de indicadores o variables y la construcción de índices, ha permitido en las Ciencias Sociales hacer observable los conceptos que a simple vista no pueden ser observables.

En la mirada de los investigadores urge la necesidad de romper con el paradigma positivista cientificista de la ciencia, impuesto por occidente que se encuentra enraizada en las preconcepciones, no solo de quienes proponen las políticas investigativas y dirigen las instituciones de educación superior, donde el pensamiento único sigue imperando, sino también en las prácticas y concepciones de investigadores de las distintas áreas del saber y desplazar la mirada hacia otros horizontes epistemológicos para construir ciencia como es lo feminista , lo ambiental, lo decolonial, la hermenéutica y el paradigma de la complejidad, evidenciando que hay otras formas de hacer investigación científica.

La aspiración positivista en investigación social es irrealizable, porque no resultaría posible cumplir con la pretensión de universalización y generalización del saber sobre los objetos de investigación propuesto por este paradigma.

Una de las estrategias efectivas para que la "Ética Socrática" empiece a tener cabida en una sociedad corrupta a la que nos enfrentamos en la actualidad en el mundo entero, donde lo común es la deshonestidad y la aplicación de la ética a medida de las conveniencias de las personas, es retomando dentro de los sistemas educativos la "Educación en Valores", así como 
el "ejemplo que los demás miembros de las organizaciones sociales debemos brindar, con lo que se mejoraría no solo procesos éticos de investigación, sino sobretodo una convivencia más digna entre los seres humanos, donde el respeto al otro, el cuidado a la naturaleza y a las otras especies que en ella conviven sea lo primordial, tendiente a construir una sociedad más humana y justa.

\section{REFERENCIAS BIBLIOGRÁFICAS:}

- Achío, M. (2003). Los Comités de Ética y la Investigación en Ciencias Sociales. Revista Ciencias Sociales 99: 85-95, 2003 (I).

- Alvarado, S.V. et. al. (2018). Polifonías del sur. Desplazamientos y desafíos de las Ciencias Sociales. Buenos Aires: Clacso.

- Alvarado, S.V (2020). Planificación de la case 1: Fundamentos epistemológicos de la investigación social contemporánea en America Latina y el Caribe, Clacso.

- Atkinson, P. et. al (2004). Manual de investigación cualitativa. Vol. IV. 32 Perspectivas analíticas.

- Bryman, A. (2006). Integrating quantitative and qualitative research: how is it done? Vol. 6 (1) $97-113$.

- Coffey, A. (2003). Encontrar el sentido a los datos cualitativos: Estrategias complementarias de investigación. Editorial Universidad de Antioquía, Colombia.

- Cohen, N. et. al, (2019). Metodología de la investigación, ¿para qué?: la producción de los datos y los diseños, Buenos Aires, Argentina.

- Cornejo, M. et. al, (2011). Desafíos en la generación de conocimiento en la investigación social cualitativa contemporánea, Revista Social Research, Volumen 12, No. 1, Art. 9.
- De Sousa Santos, B. (2008). Los desafíos de las ciencias sociales hoy. En pensar el Estado y la sociedad: desafíos actuales. Buenos Aires: Clacso.

- Lazarsfeld, Paul. (1973). "De los conceptos a los índices empíricos", en Raymond Boudon y Paul Lazarsfeld, Metodología de las ciencias sociales, Barcelona: Laia, vol. I.

- Marradi, Archenti y Piovani (2007). Metodología de las ciencias sociales; capítulo 4 el papel de la teoría en la investigación social.

- Marradi, A. y Piovani, J. I. (2002), "Los fundamentos filosóficos de los métodos de la ciencia". En DEI, D. (ed.), Pensar y hacer en investigación. Bs. As.: Docencia. SCHWARTZ, H. y JACOBS, J. (1984), Sociología cualitativa. Método para la reconstrucción de la realidad. México: Trellas. (Cap. 1: Métodos cualitativos y métodos cuantitativos: dos enfoques a la sociología).

- Piovani, J. I. (2018) "Triangulación y métodos mixtos". En A. Marradi, N. Archenti y J. I. Piovani, Manual de metodología de las ciencias sociales. Buenos Aires: Siglo Veintiuno.

- Santos, J. et. al, (2018). Métodos mixtos y reflexibidad: explorando posibles articulaciones.

- Schettini, P. et. al, (2015). Análisis de datos cualitativos en la investigación social. Procedimientos y herramientas para la interpretación de información cualitativa. Editorial de la Universidad de la Plata, Argentina.

- Stinchcombe, A. (1968), Constructing Social Theories, Nueva York, Harcourt, Brace \& World. 
- Tapia, H. (1995). La investigación científica en la educación. Ed. Grafioffset, Riobamba - Ecuador.

- Vasilachis, I. (2009). Los fundamentos ontológicos y epistemológicos de la investigación cualitativa. Revista Forum: Qualitative Social Research Volumen 10, No. 2, Art. 30. 